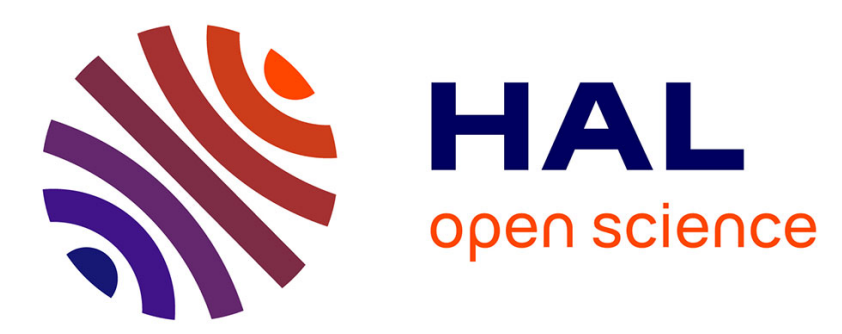

\title{
A simple correction method of inner filter effects affecting FEEM and its application to the PARAFAC decomposition
}

\author{
Xavier Luciani, Stéphane Mounier, Roland Redon, André Bois
}

\section{- To cite this version:}

Xavier Luciani, Stéphane Mounier, Roland Redon, André Bois. A simple correction method of inner filter effects affecting FEEM and its application to the PARAFAC decomposition. Chemometrics and Intelligent Laboratory Systems, 2009, 96 (2), pp.227-238. 10.1016/j.chemolab.2009.02.008 . hal00807769

\section{HAL Id: hal-00807769 \\ https://hal.science/hal-00807769}

Submitted on 4 Apr 2013

HAL is a multi-disciplinary open access archive for the deposit and dissemination of scientific research documents, whether they are published or not. The documents may come from teaching and research institutions in France or abroad, or from public or private research centers.
L'archive ouverte pluridisciplinaire HAL, est destinée au dépôt et à la diffusion de documents scientifiques de niveau recherche, publiés ou non, émanant des établissements d'enseignement et de recherche français ou étrangers, des laboratoires publics ou privés. 


\title{
A simple correction method of inner filter effects affecting FEEM and its application to the PARAFAC decomposition
}

\author{
X. Luciani ${ }^{\mathrm{a}, \mathrm{b}, *}$, S. Mounier ${ }^{\mathrm{b}}$, R. Redon ${ }^{\mathrm{b}}$, A. Bois ${ }^{\mathrm{b}}$ \\ ${ }^{a}$ Laboratoire I3S, UMR6070, UNSA CNRS, 2000, route des Lucioles, BP 121, \\ 06903 Sophia Antipolis Cedex - France \\ ${ }^{\mathrm{b}}$ Laboratoire PROTEE, USTV, BP 20132, 83957 La Garde Cedex, France
}

\begin{abstract}
In this paper we introduce a new inner filters correction method for standard fluorometer. The Controlled Dilution Approach (CDA) deals with highly absorbing solutions using the Fluorescent Excitation-Emission Matrix of a controlled weak dilution. Along with the non linear FEEM of the original solution, these two informations allow to estimate the linearized FEEM. The method relies on inner filter effects modelization. Beyond its numerical simplicity, the main interest is that CDA only requires fluorescence measurements. The method was validated using a set of known mixtures and a set of dissolved organic matter samples. In addition we show that the corrected FEEM can be used efficiently for advanced multilinear analysis. Therefore CDA is presented here as a relevant pretreatment to the PARAFAC decomposition of highly absorbing mixtures.
\end{abstract}

Key words: EEM, inner filter effects, PARAFAC, 3D fluorescence

* Corresponding autor.
Email address: lucianix@gmail.com (X. Luciani). 


\section{Introduction}

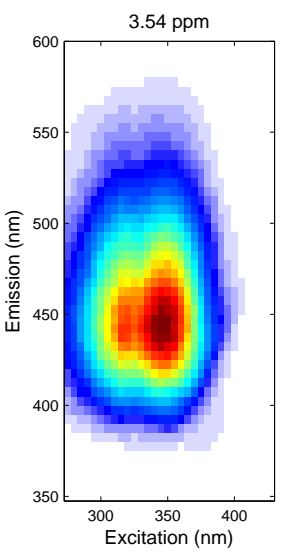

\subsection{Non linearities in fluorescence spectroscopy}

Fluorescent molecular components (fluorophores) can be easily distinguished by their spectroscopic properties and more particularly by their fluorescence spectra [1]. Recent fluorometers provide successive measurements of the fluorescence intensity emitted by a solution of one or several fluorophores. By scanning excitation and emission wavelength domains, Fluorescent ExcitationEmission Matrices (FEEM) gather a lot of informations about the solution. These spectra are now widely used in various scientific domains such as medicine [2], analytical chemistry [3] or environmental sciences $[4,5]$.

Ideally, considering the FEEM $\left(I_{3 D}\right)$ of a single fluorophore, its norm is proportional to the fluorophore concentration in the solution and its pattern is given by the outer product between the excitation spectrum and the emission spectrum of the fluorophore. This is the classical linear model of fluorescence. However it is well known that its pertinence decreases with the concentration [6]. Actually, non linear deviations mean that the gradual absorption by the solution of both exciting and fluorescent lights cannot be neglected. These effects are known as inner filter effects and affect both $I_{3 D}$ norm and $I_{3 D}$ pattern. Therefore, in presence of inner filter effects, one cannot deduce any correct information about the solution directly from $I_{3 D}$.

. Evolution of the quinine sulphate 3D spectrum for three different concentrations: $3.54 \mathrm{ppm}$ (left spectrum), $51.54 \mathrm{ppm}$ (middle spectrum), $108.75 \mathrm{ppm}$ (right spectrum)

Example of inner filter effects is given on figure 1. This example clearly shows that the FEEM pattern can be severely affected even in the simple case of a single fluorophore solution.

In other respects, considering several solutions of the same diluted fluorophore 
measured in different conditions, many other factors such as diffusion, temperature variations, $\mathrm{pH}$ variations, fluorescence quenching or ionic strength can affect the FEEM linearity [1]. In this work, we only focus on inner filter effects correction.

\subsection{Inner filter effects correction}

Inner filter effects are observed and studied for a long time now [7,8]. Two main correction methods are used to prevent these deviations. Since inner filter effects can be neglected for weak absorbances i.e. weak concentrations, a common procedure is to strongly dilute the solution until maximal absorbance is inferior than 0.1 [1]. There is an obvious drawback with this dilution method as a too strong diluting factor would severely reduce the signal to noise ratio. Moreover this procedure must be applied very carefully to avoid contamination or physico-chemical changes. Therefore, ensuring the linearity of the data set is no easy task. The second approach uses a mathematical model of inner filter effects [9-11]. Then one can deduce a correction factor in order to estimate element by element, a corrected FEEM $\left(I_{c}\right)$ from $I_{3 D}$. It is assumed that if the correction factor is suitable then $I_{c}$ will follow the linear model. This approach relies on the Beer-Lambert law [1] which gives the elementary variation $d I$ of the light intensity through an elementary optical path $d l$ at wavelength $\lambda$ :

$$
d I=-I(\lambda) \alpha(\lambda) d l
$$

where $\alpha$ is the absorption coefficient of the solution. Then, the integrated law describes the light absorption through the entire optical path. If $I_{0}$ is the intensity of the exciting light, the transmitted intensity outside a cell of length $l$ is simply given by the relation:

$$
I(\lambda)=I_{0}(\lambda) e^{-\alpha(\lambda) l}=I_{0}(\lambda) 10^{-A(\lambda)}
$$

The absorbance spectrum of the solution is then defined by

$$
A(\lambda)=\log _{10}\left(\frac{I_{0}(\lambda)}{I(\lambda)}\right)=\frac{l \alpha}{\log (10)}
$$

Figure 2 shows a schematic diagram of absorbance measurement. $A$ is obtained by measuring the transmitted intensity through the diluted solution $\left(I_{T}\right)$ and the transmitted intensity through the solvent $\left(I_{R}\right)$ at successive wavelength:

$$
A(\lambda)=\log _{10}\left(\frac{I_{R}(\lambda)}{I_{T}(\lambda)}\right)
$$

In right angle fluorescence spectroscopy, classical model of inner filter effects is given by equation 5 . 


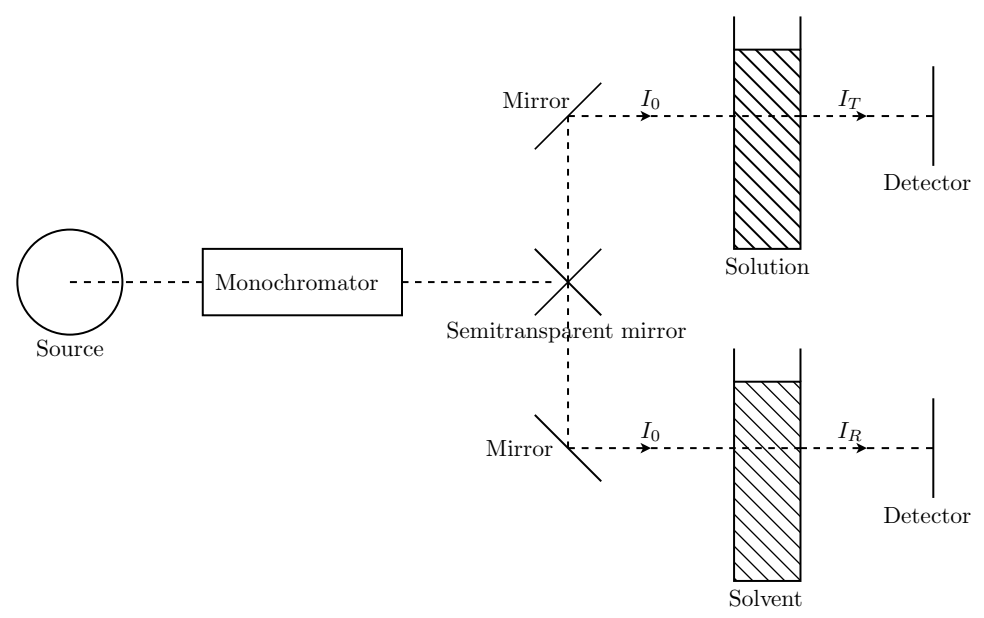

Fig. 2. Absorbance measurement

$$
I_{3 D}\left(\lambda_{e x}, \lambda_{e m}\right)=I_{c} 10^{-\frac{A\left(\lambda_{e x}\right)+A\left(\lambda_{e m}\right)}{2}}
$$

Thereby, one can use the measured absorbance spectrum to compute the correction factor and then deduce $I_{c}$. Similar methods were proposed in [12-14]. In the following, this approach will be identified as the Absorbance Correction Approach (ACA). ACA is commonly used in applicative papers dealing with fluorescence spectroscopy $[15,16]$. However absorbance measurement is much less sensitive than fluorescence measurement. In addition it requires an other experimental device whose characteristics are different, introducing its own error in the chain. Finally, the short linear range of absorption measurement is another important drawback of ACA. In this work we propose an original correction method: the Controlled Dilution Approach (CDA) which combines the advantages of both methods. CDA uses the FEEM of a diluted solution instead of absorbance measurement in order to estimate $I_{c}$. The crucial point is that the dilution factor can be chosen small enough to avoid the drawback of the dilution approach. Indeed, the linearity of this second FEEM is not required. Consequently, CDA keeps the main advantage of ACA which is a very simple numerical correction, but it only requires fluorescence spectra. Analized solutions are generally mixtures of several fluorophores. Therefore, many applications involve a separation step to recover the underlying individual spectra and concentration profiles of each fluorophore. Number of chemometric methods were proposed in the literature in order to perform multilinear decompositions of FEEM [17-20]. Based on original works of Harshman [21], PARAllel FACtor analysis (PARAFAC) was introduced in this context by Bro [22]. During the last decade PARAFAC has proved to be the most relevant approach. For instance, in environmental sciences, it is currently the reference tool to characterize and trace Dissolved Organic Matter (DOM) [23-25]. In return, it do not take into account inner filter effects [26,27]. Consequently there is an irreversible loss of performance when dealing with highly absorbing mixtures. 
Like other inner filter effects correction methods, CDA is independent of this separation step. However, we take into consideration that a large part of FEEM applications, uses this kind of decomposition. As a consequence, in order to ensure the reliability of CDA, we also present in this paper its performance as a PARAFAC pretreatment of highly absorbing mixtures.

\subsection{Paper organization}

CDA is detailed on section 2 of this paper. First the modelization of inner filter effects is given in section 2.1 then CDA is described on section 2.2. Lastly, practical aspects of CDA are presented in section 2.3 notably in the case of FEEM sets analysis. The PARAFAC application to the CDA corrected FEEM is shortly describe.

In this work, CDA correction is experimentally tested on two very different sets of mixtures. The first set is composed of standard laboratory mixtures of fluorescein and quinine sulphate. Consequently, thist first data set is used to strictly validate CDA and compare with classical ACA. On the other side, the second data set is constituted by unknown samples of DOM catchments and gives an example of how the method can help in a realistic case. Section 3 describes the experimental part of these tests. Results obtained on both data sets before and after the PARAFAC decomposition are presented and discussed in section 4 .

\section{Theory}

\subsection{Modelization of inner filter effects}

Like ACA, CDA relies on equation 5. Few authors give detailed mathematical justifications of this model, particularly in the most general case of 3D spectra of fluorophore mixtures. In this subsection a rigorous interpretation of equation 5 is proposed.

We consider here a mixture of $N$ fluorophores. For each fluorophore $n$, we note $c_{n}$ its concentration in the solution, $\varepsilon_{n}\left(\lambda_{e x}\right)$ its molar extinction coefficient at the excitation wavelength $\lambda_{e x}, \Phi_{n}$ its the quantum yield of fluorophore $n, \gamma_{n}\left(\lambda_{e m}\right)$ its emission probability at wavelength $\lambda_{e m}$ and $\alpha_{n}\left(\lambda_{e x}\right)$ its absorption coefficient which is equal to the product of $c_{n}$ by $\varepsilon_{n}\left(\lambda_{e x}\right)$. We assume that the absorption and emission spectra of fluorophore $n$ are normalized values of respectively $\varepsilon_{n}\left(\lambda_{e x}\right)$ and $\gamma_{n}\left(\lambda_{e m}\right)$. In the linear approximation, every fluorescing particles are treated equally as if the whole sample cell was an ele- 
Sample cell the particular geometry of the problem.
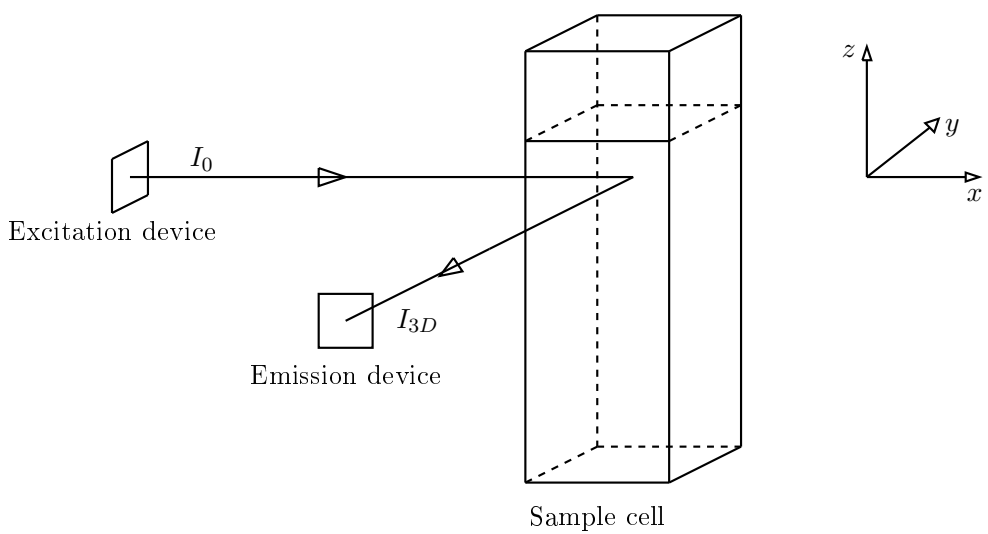

Fig. 3. Schematic diagram of right angle fluorescence measurement

mentary point. In order to improve this model, one should takes into account

Figure 3 recalls basically the experimental device of right angle standard fluorometers. The excitation light $\left(I_{0}\left(\lambda_{e x}\right)\right)$ is absorbed through the sample cell (length $l$ ) by the fluororphores, inducing the fluorescent light. Finally, a fraction $\left(I_{3 D}\left(\lambda_{e m}\right)\right)$ of the emitted signal is collected perpendicularly to the exciting beam. $\lambda_{e x}$ and $\lambda_{e m}$ scannings allow to measure the FEEM.

In this study, several approximations were made. First of all, we took into consideration the symmetry of the problem, therefore the influence of the $z$ spatial dimension was neglected.

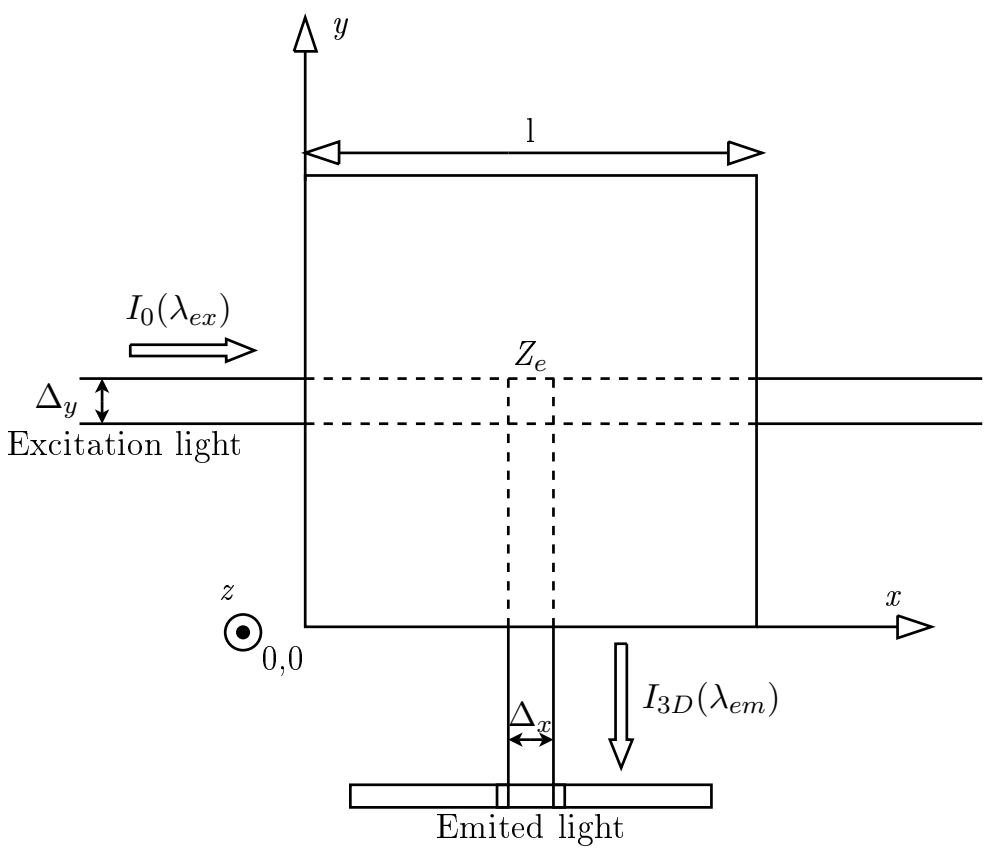

Fig. 4. Scheme of the sample cell, view from above 
Secondly, only two main optical paths were considered. They represent the excitation beam and emission beam in figure 4 scheme. This means that the fraction of the exciting light which do not reach the "influence zone" $Z_{e}$ was neglected as well as the fluorescence light issued from the region outside $Z_{e}$. Then each elementary segment of the "excited face" of $Z_{e}$ was supposed to receive the same energy from the rectilinear exciting beam. In the same way, we assumed that each elementary segment of the "emission face" of $Z_{e}$ provides the same energy to the detector. Furthermore, diffusion and re-emission effects were also neglected. Actually we only consider the elementary optical paths represented in figure 5 .

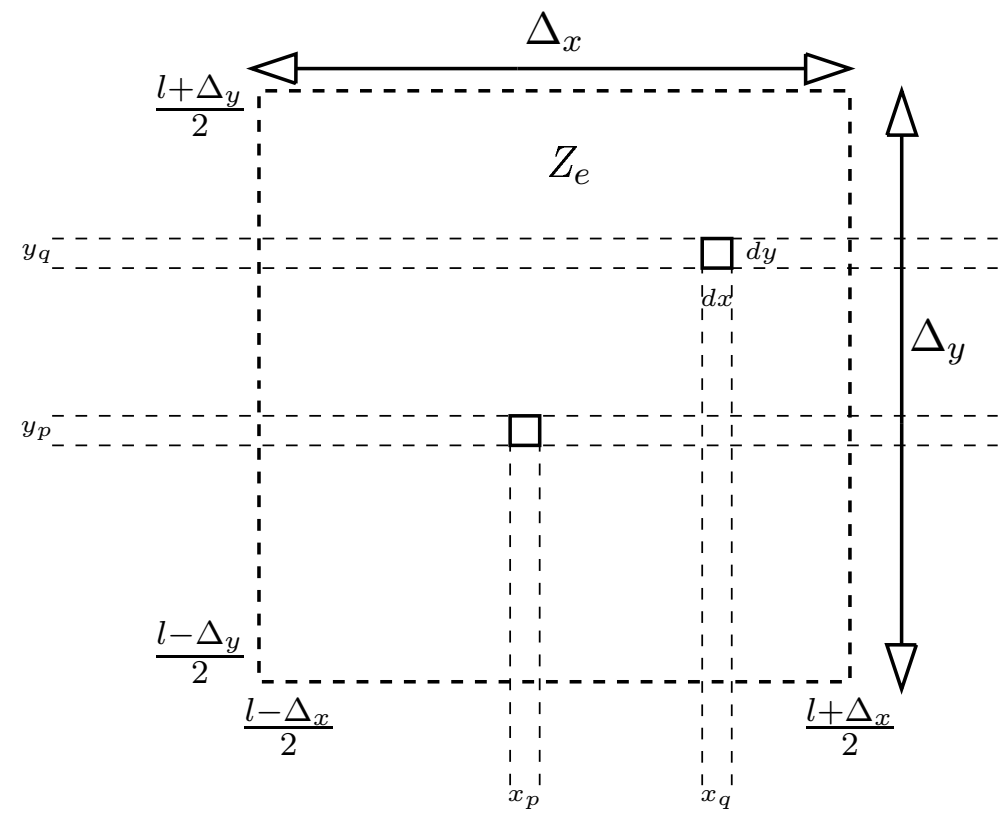

Fig. 5. Elementary cutting of the "influence zone" in the directions of exciting and emitted ligthts

The integrated Beer-Lambert law describes the light absorption through the optical path. If $I_{0}$ is the light intensity at the point $x_{0}$ of the dilute solution, the intensity in $x$ is simply given by the relation: $I=I_{0} e^{-\alpha\left(\lambda_{e x}\right)\left(x-x_{0}\right)}$ where $\alpha$ is the mixture total absorption coefficient: $\alpha=\sum_{n} \alpha_{n}$. The influence zone was divided into horizontal and vertical elementary strips of respective dimension $d y \times l$ and $l \times d x$. Then each horizontal strip receives an equal elementary fraction of the exciting light: $\frac{d y I_{0}}{\Delta_{y}}$ and the Beer-Lambert law quantifies the intensity transmitted to $x$. A fraction $\alpha_{n} d x$ is absorbed by fluorophore $n$, and the total intensity absorbed by fluorophore $n$ in the "influence zone" $\left(I_{A n}\right)$ is given by:

$$
I_{A n}\left(\lambda_{e x}\right)=\frac{d y I_{0}}{\Delta_{y}} \alpha_{n}\left(\lambda_{e x}\right) \int_{\frac{l-\Delta_{x}}{2}}^{\frac{l+\Delta_{x}}{2}} e^{-\alpha\left(\lambda_{e x}\right) x} d x
$$




$$
I_{A n}\left(\lambda_{e x}\right)=2 \frac{I_{0}}{\Delta_{y} \alpha\left(\lambda_{e x}\right)} \alpha_{n}\left(\lambda_{e x}\right) e^{-\frac{\alpha\left(\lambda_{e x}\right) l}{2}} \sinh \left(\frac{\alpha\left(\lambda_{e x}\right) \Delta_{x}}{2}\right) d y
$$

The fluorescence signal emitted by the $d y$ strip at wavelength $\lambda_{e m}$ is equal to $\sum_{n} \Phi_{n} \gamma_{n}\left(\lambda_{e m}\right) I_{A n}\left(\lambda_{e x}\right)$ and the Beer-Lambert law integrated on all the elementary horizontal strips gives the ratio of the fluorescence signal transmitted outside the sample cell in the $y$ direction, $I_{3 D}\left(\lambda_{e x}, \lambda_{e m}\right)$.

$$
\begin{gathered}
I_{3 D}\left(\lambda_{e x}, \lambda_{e m}\right)=\int_{\frac{l-\Delta_{y}}{2}}^{\frac{l+\Delta_{y}}{2}} \sum_{n} \Phi_{n} \gamma_{n}\left(\lambda_{e m}\right) I_{A n}\left(\lambda_{e x}\right) e^{-\alpha\left(\lambda_{e m}\right) y} \\
I_{3 D}\left(\lambda_{e x}, \lambda_{e m}\right)=\frac{4 I_{0} e^{-\frac{\alpha\left(\lambda_{e x}\right) l}{2}} \sinh \left(\frac{\alpha\left(\lambda_{e x}\right) \Delta_{x}}{2}\right) e^{-\frac{\alpha\left(\lambda_{e m}\right) l}{2}} \sinh \left(\frac{\alpha\left(\lambda_{e m}\right) \Delta_{y}}{2}\right)}{\Delta_{y} \alpha\left(\lambda_{e x}\right) \alpha\left(\lambda_{e m}\right)} \sum_{n} \alpha_{n}\left(\lambda_{e x}\right) \Phi_{n} \gamma_{n}\left(\lambda_{e m}\right)
\end{gathered}
$$

$\frac{\alpha\left(\lambda_{e x}\right) \Delta_{x}}{2}$ and $\frac{\alpha\left(\lambda_{e m}\right) \Delta_{y}}{2}$ are supposed to be small enough to make the following approximation:

$$
I_{3 D}\left(\lambda_{e x}, \lambda_{e m}\right)=I_{0} \Delta_{x}\left(\sum_{n} \alpha_{n}\left(\lambda_{e x}\right) \Phi_{n} \gamma_{n}\left(\lambda_{e m}\right)\right) e^{-\frac{\alpha\left(\lambda_{e x}\right) l}{2}} e^{-\frac{\alpha\left(\lambda_{e m}\right) l}{2}}
$$

Then we can define $g=\frac{l}{2}$ and $G_{n}=I_{0} \Delta_{x} \Phi_{n}$. This leads to the final expression of the model:

$$
I_{3 D}\left(\lambda_{e x}, \lambda_{e m}\right)=\left(\sum_{n=1}^{N} G_{n} c_{n} \varepsilon_{n}\left(\lambda_{e x}\right) \gamma_{n}\left(\lambda_{e m}\right)\right) \prod_{n=1}^{N} e^{-g\left(c_{n} \varepsilon_{n}\left(\lambda_{e x}\right)+c_{n} \varepsilon_{n}\left(\lambda_{e m}\right)\right)}
$$

In the following we define

$$
L\left(\lambda_{e x}, \lambda_{e m}\right)=\sum_{n=1}^{N} G_{n} c_{n} \varepsilon_{n}\left(\lambda_{e x}\right) \gamma_{n}\left(\lambda_{e m}\right)
$$

then we have

$$
I_{3 D}\left(\lambda_{e x}, \lambda_{e m}\right)=L e^{-g\left(c_{n} \varepsilon_{n}\left(\lambda_{e x}\right)+c_{n} \varepsilon_{n}\left(\lambda_{e m}\right)\right)}
$$

This equation is clearly equivalent to equation 5 with $I_{c}=L$. Its first order approximation is justified for small enough concentrations. In this case, since the exponential term tends to 1 one obtains the linear model of fluorescence. Correction of inner filter effects simplifies spectral analysis. It is interesting to note that their modelization is also used in another context. Actually, a recent article [28] highlighted the major contribution of inner filter effects in the phenomenon of concentration-dependent red-shift [29,30]. In this work, a similar model has been successfully used to optimize synchronous fluorescence spectroscopy of concentrated mixtures of fluorophores. 


\subsection{Controlled Dilution Approach}

The previous model describes non linear effects but the related equation can still be considered as a bilinear decomposition involving some modified individual spectra $\varepsilon_{n}^{\prime}\left(\lambda_{e x}\right)$ and $\gamma_{n}^{\prime}\left(\lambda_{e m}\right)$ :

$$
I_{3 D}\left(\lambda_{e x}, \lambda_{e m}\right)=\sum_{n=1}^{N} \varepsilon_{n}^{\prime}\left(\lambda_{e x}\right) \gamma_{n}^{\prime}\left(\lambda_{e m}\right)
$$

with,

$$
\begin{gathered}
\varepsilon_{n}^{\prime}\left(\lambda_{e x}\right)=G_{n} c_{n} \varepsilon_{n}\left(\lambda_{e x}\right) e^{-\sum_{p=1}^{N} g c_{p} \varepsilon_{p}\left(\lambda_{e x}\right)} \\
\gamma_{n}^{\prime}\left(\lambda_{e m}\right)=\gamma_{n}\left(\lambda_{e m}\right) e^{-\sum_{p=1}^{N} g c_{p} \varepsilon_{p}\left(\lambda_{e m}\right)}
\end{gathered}
$$

It is well known that bilinear decompositions have an infinite number of equivalent solutions in the least square sense. Therefore, without additional information, no mathematical tool can diagnose whether a FEEM is affected by inner filter effects or not. A fortiori additional informations are also needed to correct inner filter effects. In ACA this information is the solution absorbance spectrum. This section shows how the correction can be made with fluorescent spectra only.

According to equation 11 the FEEM $I_{3 D}$ of a $N$ fluorophores mixture is the product of a linear $(L)$ term in respect of concentrations and spectra by a non linear one, denoted $H$ :

$$
H\left(\lambda_{e x}, \lambda_{e m}\right)=\prod_{n=1}^{N} \exp \left(-g\left(c_{n} \varepsilon_{n}\left(\lambda_{e x}\right)+c_{n} \varepsilon_{n}\left(\lambda_{e m}\right)\right)\right.
$$

So we can write:

$$
I_{3 D}\left(\lambda_{e x}, \lambda_{e m}\right)=L\left(\lambda_{e x}, \lambda_{e m}\right) H\left(\lambda_{e x}, \lambda_{e m}\right)
$$

Now, let's $I_{3 D_{p}}$ be the FEEM of the same mixture, diluted by a factor $p$, then we have:

$$
\begin{gathered}
I_{3 D p}\left(\lambda_{e x}, \lambda_{e m}\right)=\left(\sum_{n=1}^{N} G_{n} \frac{c_{n}}{p} \varepsilon_{n}\left(\lambda_{e x}\right) \gamma_{n}\left(\lambda_{e m}\right)\right) \prod_{n=1}^{N} e^{-g\left(\frac{c_{n}}{p} \varepsilon_{n}\left(\lambda_{e x}\right)+\frac{c_{n}}{p} \varepsilon_{n}\left(\lambda_{e m}\right)\right)} \\
I_{3 D p}\left(\lambda_{e x}, \lambda_{e m}\right)=\frac{1}{p} L\left(\lambda_{e x}, \lambda_{e m}\right) H^{\frac{1}{p}}\left(\lambda_{e x}, \lambda_{e m}\right)
\end{gathered}
$$

The analytical resolution of 18 and 20 gives

$$
\begin{aligned}
L\left(\lambda_{e x}, \lambda_{e m}\right) & =\left(\frac{\left(p I_{3 D p}\left(\lambda_{e x}, \lambda_{e m}\right)\right)^{p}}{I_{3 D}\left(\lambda_{e x}, \lambda_{e m}\right)}\right)^{\frac{1}{p-1}} \\
H\left(\lambda_{e x}, \lambda_{e m}\right) & =\left(\frac{I_{3 D}\left(\lambda_{e x}, \lambda_{e m}\right)}{p I_{3 D p}\left(\lambda_{e x}, \lambda_{e m}\right)}\right)^{\frac{p}{p-1}}
\end{aligned}
$$


The $L$ term is the corrected FEEM estimated by CDA, corresponding to the linear model of fluorescence. As previously mentioned, the correction only requires the original FEEM and the diluted FEEM and the value of the dilution factor $p$.

The sensitivity of the estimator of $L$ to $p$ is difficult to quantify. A first order approximation of the variability of $L\left(\Delta_{L}\right)$ leads to:

$$
\frac{\Delta_{L}}{L}=\frac{\left(p-1-\log (p)-\log \left(I_{3 D p}\right)+\log \left(I_{3 D}\right)\right) \Delta_{p}}{(p-1)^{2}}
$$

According to this equation, a high factor should be preferred. However it would involve the drawbacks of a strong dilution (see section 1.2). Finally, we advocate for a dilution factor corresponding to the simplest dilution process, thus the experimental uncertainty $\Delta_{p}$ is minimized. This was the case for all the experiments presented in this study.

Owing to the term by term division in equation 21, noisy values in the measured FEEM could affect the estimation of $L$. Actually if the division involves two small values relatively to the noise level, some very narrow and localized peaks can appear. Fluorescent spectroscopy is a very sensitive technique therefore this kind of deviation are rarely observed in practical situations. Otherwise those peaks appear outside the main fluorescing areas. In consequence, they can be easily detected and filtered without damaging the fluorescent peaks.

\subsection{CDA and multilinear analysis of concentrated fluorescing mixtures}

We consider now a set of $I$ mixtures and $c_{n}(i)$ denotes the concentration of fluorophore $n$ in mixture $i$. CDA methodology is simple, the correction is done sample by sample. The first step consists in choosing the dilution factor $p$ for each sample (see the end of section 2.2). Obviously the same value can be used for every sample. Then, the corresponding controlled dilution is performed and both FEEM $I_{3 D}$ and $I_{3 D p}$ are measured. Before correction, Rayleigh and Raman scatters must be corrected carefully on each FEEM. This is the end of the experimental and pre-processing steps.

Finally, for each sample $i$, the estimation $L\left(i, \lambda_{e x}, \lambda_{e m}\right)$ of the linearized FEEM is obtained directly from equation 21. At this stage, the correction of the inner filter effects is completed.

Actually we have to take into account measurement and modelization errors. In addition we can define $\tilde{c}_{n}(i)=G_{n} c_{n}(i)$. Therefore in practice, definition 12 is rewritten:

$$
L\left(i, \lambda_{e x}, \lambda_{e m}\right)=\sum_{n=1}^{N} \tilde{c}_{n}(i) \varepsilon_{n}\left(\lambda_{e x}\right) \gamma_{n}\left(\lambda_{e m}\right)+E\left(i, \lambda_{e x}, \lambda_{e m}\right)
$$


where $E$ is the error term. Equation 23 is a rank $N$ decomposition of the 3 way tensor $L$ or in other words a 3 -way PARAFAC model of rank $N$. For each fluorophore $n$, the loading vectors of the decomposition $\tilde{c}_{n}, \varepsilon_{n}$, and $\gamma_{n}$ are linearly linked to its concentration profile, its excitation spectrum and its emission spectrum respectively. Moreover, the solution of this decomposition is unique up to trivial scaling and position indeterminacy [31,32]. Finally, several efficient algorithms were proposed and compared for the estimation of the loading vectors. These are largely described in the literature [33-35]. Those three physical, mathematical and practical reasons made the PARAFAC decomposition the most suitable tool for analysing linear(ized) FEEM. Tutorials and examples of PARAFAC application to FEEM analysis can be found elsewhere $[36,22,37]$.

Eventually, the PARAFAC decomposition can be run normally on the corrected FEEM set in order to find out real individual spectra and concentration profiles of each fluorophore.

\section{Experimental}

\subsection{Data set 1, standard mixtures}

Seven solutions $\left(S_{1}^{i}, i=1 \cdots 7\right)$ with different concentrations of fluorescein (Aldrich) and quinine sulphate (Merkc) were prepared in $0.1 \mathrm{M} \mathrm{H}_{2} \mathrm{SO}_{4}$ (Aldrich) in order to validate the correction method. All chemicals are analytical grade. Concentrations in fluorescein and quinine sulphate are given in table 1 along with solution absorbances. These two fluorophores and their concentrations in the solutions were chosen because of their good fluorescing ability and their overlapped spectra in order to emphasize inner filter effects.

Table 1

Concentrations, maximal absorbances and mean absorbances of the original solutions of quinine sulphate and fluorescein.

\begin{tabular}{cccccccc}
\hline Solution & $\mathrm{S}_{1}^{1}$ & $\mathrm{~S}_{1}^{2}$ & $\mathrm{~S}_{1}^{3}$ & $\mathrm{~S}_{1}^{4}$ & $\mathrm{~S}_{1}^{5}$ & $\mathrm{~S}_{1}^{6}$ & $\mathrm{~S}_{1}^{7}$ \\
\hline$c_{S Q}(\mathrm{ppm})$ & 0 & 11.02 & 32.6 & 54.38 & 76.15 & 97.73 & 108.75 \\
$c_{F}(\mathrm{ppm})$ & 83.15 & 74.72 & 58.23 & 41.58 & 24.92 & 8.43 & 0 \\
Absorbance max. & 2.30 & 2.18 & 1.83 & 1.32 & 1.17 & 1.38 & 1.47 \\
Mean Absorbance. & 0.42 & 0.40 & 0.37 & 0.33 & 0.29 & 0.27 & 0.24
\end{tabular}

Concentrations in quinine sulphate $\left(c_{S Q}\right)$ and fluorescein $\left(c_{F}\right)$ are given in parts per million (ppm). Maximum and mean value of absorbance are relative to the 275 to $500 \mathrm{~nm}$ excitation range. 
Seven twice diluted solutions $\left(S_{1 D}^{i}, i=1 \cdots 7\right)$ were obtained by mixing equal volumes of initial solutions $S_{1}^{i}$ and $0.1 \mathrm{M}$ of $\mathrm{H}_{2} \mathrm{SO}_{4}$. Table 2 gives the actual value of the dilution factor for the seven solutions and the standard deviation due to the pipet precision.

Table 2

Dilution factors used for the seven solutions of fluorescein and quinine sulphate.

\begin{tabular}{cccccccc}
\hline Solution & $\mathrm{S}_{1 D}^{1}$ & $\mathrm{~S}_{1 D}^{2}$ & $\mathrm{~S}_{1 D}^{3}$ & $\mathrm{~S}_{1 D}^{4}$ & $\mathrm{~S}_{1 D}^{5}$ & $\mathrm{~S}_{1 D}^{6}$ & $\mathrm{~S}_{1 D}^{7}$ \\
\hline$p$ & 2.11 & 2.08 & 2.07 & 2.07 & 2.07 & 2.08 & 2.11 \\
$\sigma_{p}$ & 0.034 & 0.032 & 0.030 & 0.029 & 0.030 & 0.032 & 0.034
\end{tabular}

$\overline{\sigma_{p}}$ is the estimated standard deviation of the dilution factor due to the experimental dilution

Reference solutions $\left(S_{1 R}^{i}, i=1 \cdots 7\right)$ were obtained by diluting $100 \mu \mathrm{L}$ of $S_{1}^{i}$ in $3000 \mu \mathrm{L}$ of $0.1 \mathrm{M}$ of $\mathrm{H}_{2} \mathrm{SO}_{4}$. In this case of simple mixtures, this dilution prevents inner filter effects without physico-chemical changes.

All measured spectra were obtained with a fluorometer Hitachi F4500. FEEM of the three solutions sets $S_{1}, S_{1 D}$ and $S_{1 R}$, were recorded at $30000 \mathrm{~nm} / \mathrm{min}$ scan speed from 350 to $700 \mathrm{~nm}$ in emission by step of $5 \mathrm{~nm}$ and for excitation wavelength from 275 to $500 \mathrm{~nm}$ by step of $5 \mathrm{~nm}$. Excitation and emission bandwidth were $5 \mathrm{~nm}$. Fluorescence intensity was corrected from PM response using manufacturer setting. Data for FEEM treatment were extracted by FLWinLab software for emission and excitation range stepped every $5 \mathrm{~nm}$. Rayleigh and Raman scatters were removed numerically by the method proposed by Zepp in [38]. In the following, measured FEEM from original, diluted and reference solution $i$ will be referred as $I_{1 U}^{i}, I_{1 D}^{i}$ and $I_{1 R}^{i}$ respectively. Absorption spectra of solutions $S_{1}$ were obtained from transmittance spectra recorded with absorbance mode of the F4500 (speedscan $240 \mathrm{~nm} / \mathrm{min}$ ) from 200 to $800 \mathrm{~nm}$ with $5 \mathrm{~nm}$ bandwidths in excitation and emission. 2D reference spectra of fluorescein and quinine sulphate were recorded from $S_{1 R}^{1}$ and $S_{1 R}^{7}$ respectively, at $240 \mathrm{~nm} / \mathrm{min}$ scan speed by step of $1 \mathrm{~nm}$ with $5 \mathrm{~nm}$ bandwidth in excitation and $2.5 \mathrm{~nm}$ bandwidth in emission. Quinine sulphate $\left(I_{S Q-e x}\right)$ and fluorescein $\left(I_{F-e x}\right)$ excitation spectra were recorded from 275 to $500 \mathrm{~nm}$ at $450 \mathrm{~nm}$ and $510 \mathrm{~nm}$ emission wavelength respectively. Their emission spectra $\left(I_{S Q-e m}\right.$ and $\left.I_{F-e m}\right)$ were recorded from 350 to $700 \mathrm{~nm}$ at $340 \mathrm{~nm}$ and $440 \mathrm{~nm}$ excitation wavelength respectively.

$I_{1 R}$ and $I_{1 U}$ compose the groups of reference and uncorrected FEEM respectively. Using absorption spectra in equation 5, we could compute the ACA corrected FEEM $\left(I_{1 A C A}^{i}\right)$ from $I_{1 U}^{i}, i=1 \cdots 7$. In the same way, using $I_{1 D}^{i}$ in equation 21 with dilution factor values of table 2 , we could apply CDA to $I_{1 U}^{i}$ and compute the CDA corrected FEEM $\left(I_{1 C D A}^{i}\right), i=1 \cdots 7 . I_{1 A C A}$ and $I_{1 C D A}$ compose the groups of ACA and CDA corrected FEEM respectively.

These four groups of FEEM are considered as four 3-way tensors of dimensions $7 \times 46 \times 51$. Trilinear decompositions of these tensors were performed with the PARAFAC-ALS algorithm of the nway toolbox for Matlab [39]. 


\subsection{Data set 2, unknown samples}

The second data set is composed of FEEM obtained from eleven samples of concentrated humic acid solutions which were extracted from catchments of Cameron soils. For each sample, $1 \mathrm{~g}$ of soil was extracted by $30 \mathrm{~mL}$ of $\mathrm{HCl}(1 \mathrm{M})$ solution. After separation, the supernatant solution was cleaned on $\mathrm{XAD}-8$ resin and stored at $4^{\circ} \mathrm{C}$ in dark. The resting soil was then extracted with $30 \mathrm{~mL}$ of $1 \mathrm{M} \mathrm{NaOH}$ solution. After separation, this second supernatant contains humic acid substance. Purification was done by acidic precipitation and sodic redissolution. Humic acid gave dark brown solution and fulvic acid yellow solution. Original solutions $\left(S_{2}^{i}, i=1 \cdots 11\right)$, were obtained by diluting $100 \mu \mathrm{L}$ of the extracted solutions in $3000 \mu \mathrm{L}$ of $0.1 \mathrm{M} \mathrm{NaOH}$ buffer. All chemicals are analytical grade. Eleven twice diluted solutions in $0.1 \mathrm{M} \mathrm{NaOH}$ buffer $\left(S_{2 D}^{i}, i=1 \cdots 11\right)$ were also prepared. Finally, reference solutions without inner filter effect $\left(S_{2 R}^{i}, i=1 \cdots 11\right)$ were obtained by 15 times dilutions of $S_{2 D}^{i}$.

FEEM of $S_{2}^{i}\left(I_{2 U}^{i}\right), S_{2 D}^{i}\left(I_{2 D}^{i}\right)$ and $S_{2 R}^{i}\left(I_{2 R}^{i}\right)$ were recorded for $i=1 \cdots 11$, at $30000 \mathrm{~nm} / \mathrm{min}$ scan speed from 340 to $650 \mathrm{~nm}$ in emission for excitation wavelength from 240 to $600 \mathrm{~nm}$, by emission and excitation step of $5 \mathrm{~nm}$ and with $10 \mathrm{~nm}$ bandwidth in excitation and $5 \mathrm{~nm}$ bandwidth in emission. Rayleigh and Raman scatters were consistently corrected [38].

$I_{2 R}$ and $I_{2 U}$ compose the groups of reference and uncorrected FEEM respectively. Using $I_{2 D}^{i}$ in equation 21 with $p=2$, we could apply CDA to $I_{2 U}^{i}$ and compute the CDA corrected FEEM $\left(I_{2 C D A}^{i}\right), i=1 \cdots 11 . I_{2 C D A}$ compose the group of CDA corrected FEEM. Comparison with ACA was not made on this data set.

Hence, three tensors of dimensions $11 \times 73 \times 63$ were obtained and decomposed by PARAFAC-ALS [39].

\section{Results and discussion}

In the following the PARAFAC applications to uncorrected, reference, ACA corrected and CDA corrected groups of FEEM will be referred as U-PARAFAC, R-PARAFAC, ACA-PARAFAC and CDA-PARAFAC respectively.

\subsection{Data set 1}

Each CDA corrected FEEM $\left(I_{1 C D A}^{i}, i=1 \cdots 7\right)$ are firstly compared to $I_{1 R}^{i}$, $I_{1 U}^{i}$ and $I_{1 A C A}^{i}$. Representative examples of these FEEM are presented on figure 6 for $i=\{1,3,4,6\}$. In these examples, CDA provides satisfying estimations 
of the reference FEEM in spite of a small distortion in the fluorescein peak $(440 / 510 \mathrm{~nm})$, particularly on $I_{1 C D A}^{1}$. In order to quantify these comparisons three relative squared residual error terms $\left(r_{1 C D A}^{i}, r_{1 U}^{i}\right.$ and $\left.r_{1 A C A}^{i}\right)$ were computed as follow for each solution $i$ and stored in table 3 .

$$
\begin{aligned}
r_{1 C D A}^{i} & =\frac{\sum_{j, k}\left(I_{1 R}^{i}(j, k)-I_{1 C D A}^{i}(j, k)\right)^{2}}{\sum_{i, j} I_{1 R}^{i}(j, k)^{2}} \\
r_{1 U}^{i} & =\frac{\sum_{j, k}\left(I_{1 R}^{i}(j, k)-I_{1 U}^{i}(j, k)\right)^{2}}{\sum_{j, k} I_{1 R}^{i}(j, k)^{2}} \\
r_{1 A C A}^{i} & =\frac{\sum_{j, k}\left(I_{1 R}^{i}(j, k)-I_{1 A C A}^{i}(j, k)\right)^{2}}{\sum_{j, k} I_{1 R}^{i}(j, k)^{2}}
\end{aligned}
$$

The significance of the non linear term in equation (11) increases with the Table 3

Comparison of the relative squared residual error terms(in \% ) for the seven solution of data set 1.

\begin{tabular}{cccccccc}
\hline Solution & 1 & 2 & 3 & 4 & 5 & 6 & 7 \\
$r_{1 U}$ & 117 & 109 & 81 & 46 & 29 & 20 & 13 \\
$r_{1 A C A}$ & 31 & 38 & 59 & 33 & 14 & 7 & 8 \\
$r_{1 C D A}$ & 11 & 5 & 2 & 1.8 & 0.3 & 0.06 & 0.03 \\
\hline
\end{tabular}

solution absorbance. Therefore comparing tables 1 and 3, there is an obvious correlation between $r_{1 U}$ and the mean absorbances as both values decrease regularly from solutions 1 to 7 . The same observation holds true regarding $r_{1 C D A}$. The correlation is less apparent for $r_{1 A C A}$ but globally the error is greater for solutions 1 to 4 than for the least absorbing solutions. $r_{1 U}$ values are comprised between $13 \%$ and $117 \%$. After CDA correction, these boundary values decreased to $0.03 \%$ and $11 \%$ respectively . These results are very satisfying for solutions 2 to 7 . The first solution shows a stronger error but there is still a clear improvement in comparison of the original FEEM. CDA results are always clearly better than ACA ones. Actually, in the more favourable case in respect to ACA results (solution 1), $r_{1 A C A}$ is almost 3 times greater than $r_{1 C D A}$.

These results showed that CDA provided a better estimation of $I_{1 R}$ than ACA. In order to verify if CDA correction is satisfying for further analysis, the PARAFAC-ALS algorithm was applied to the four groups of FEEM. For each group, the core consistency diagnostic (CORCONDIA) [40] suggested two as the right number of components. Consequently, each PARAFAC decomposition provides an estimation of the quinine sulphate and fluorescein excitation and emission spectra and an estimation of their relative concentrations trough the data set. After normalisation, the relative squared error $\left(r^{c}, r^{e x}\right.$ and $\left.r^{e m}\right)$ between the PARAFAC loadings and the real variables are compared on tables 4 to 6 . In the case of the spectral loadings, two other spectroscopic criteria are also used: the relative error to the maximum value of the 

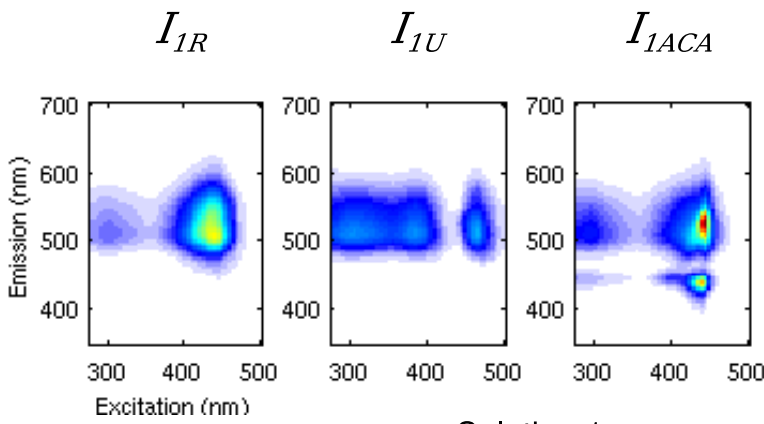

$I_{1 C D A}$

Solution 1
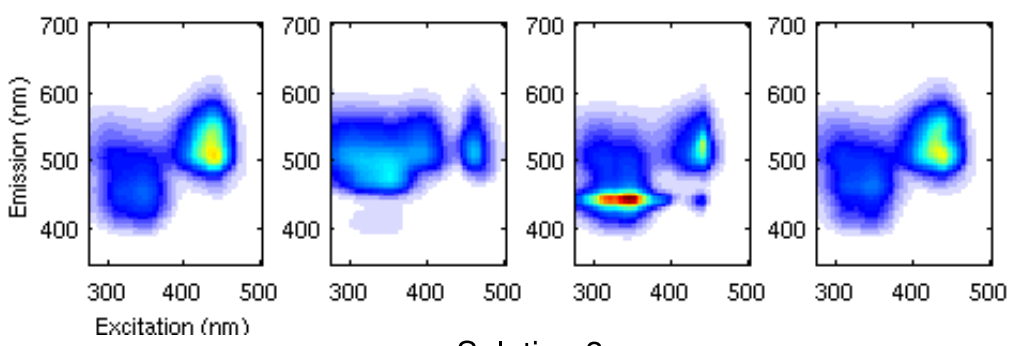

Solution 3
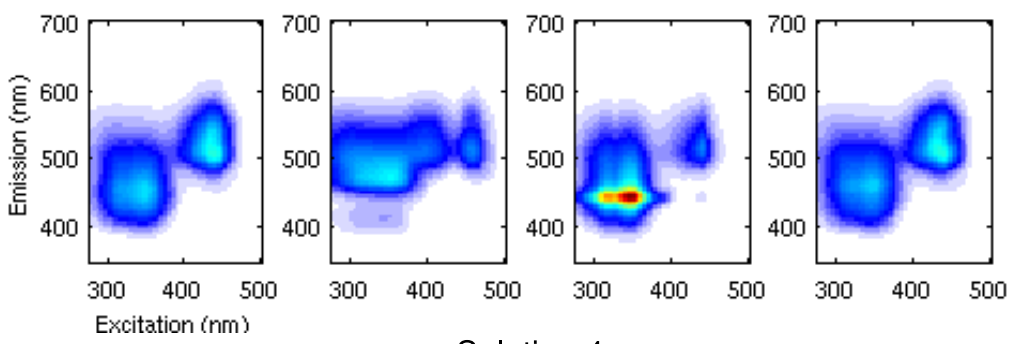

Solution 4
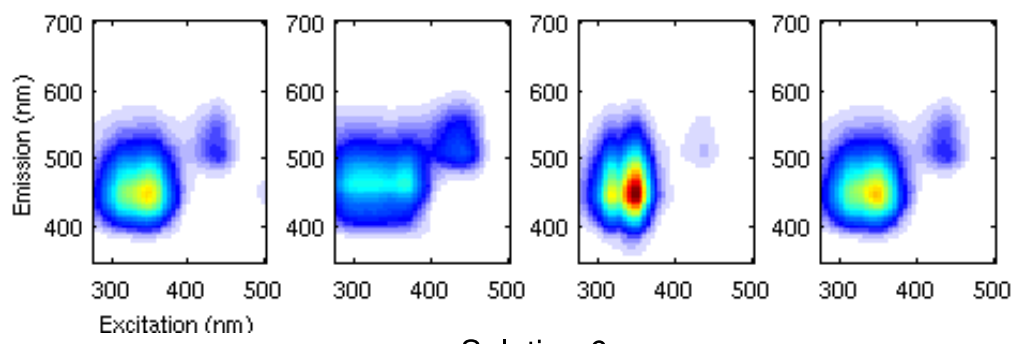

Solution 6

Fig. 6. Illustrations of $I_{1 R}, I_{1 U}, I_{1 A C A}$ and $I_{1 C D A}$ for solutions $1,3,4$ and 6 of data set 1 .

spectrum and the shift on the position of the maximum. In addition, loadings obtained from U-PARAFAC and CDA-PARAFAC are shown on figures 7 to 9, along with real spectra and profiles. A first global remark should be made: the perfect agreement on the three modes between the real variables and R-PARAFAC loadings demonstrates that estimation errors of U-PARAFAC, 


\section{Table 4}

Data set 1 , concentration mode results. Relative squared residual error in percent between the real profiles and their estimation from $I_{1 R}\left(r_{1 R}^{c}\right), I_{1 U}\left(r_{1 U}^{c}\right), I_{1 A C A}$ $\left(r_{1 A C A}^{c}\right)$ and $I_{1 C D A}\left(r_{1 C D A}^{c}\right)$.

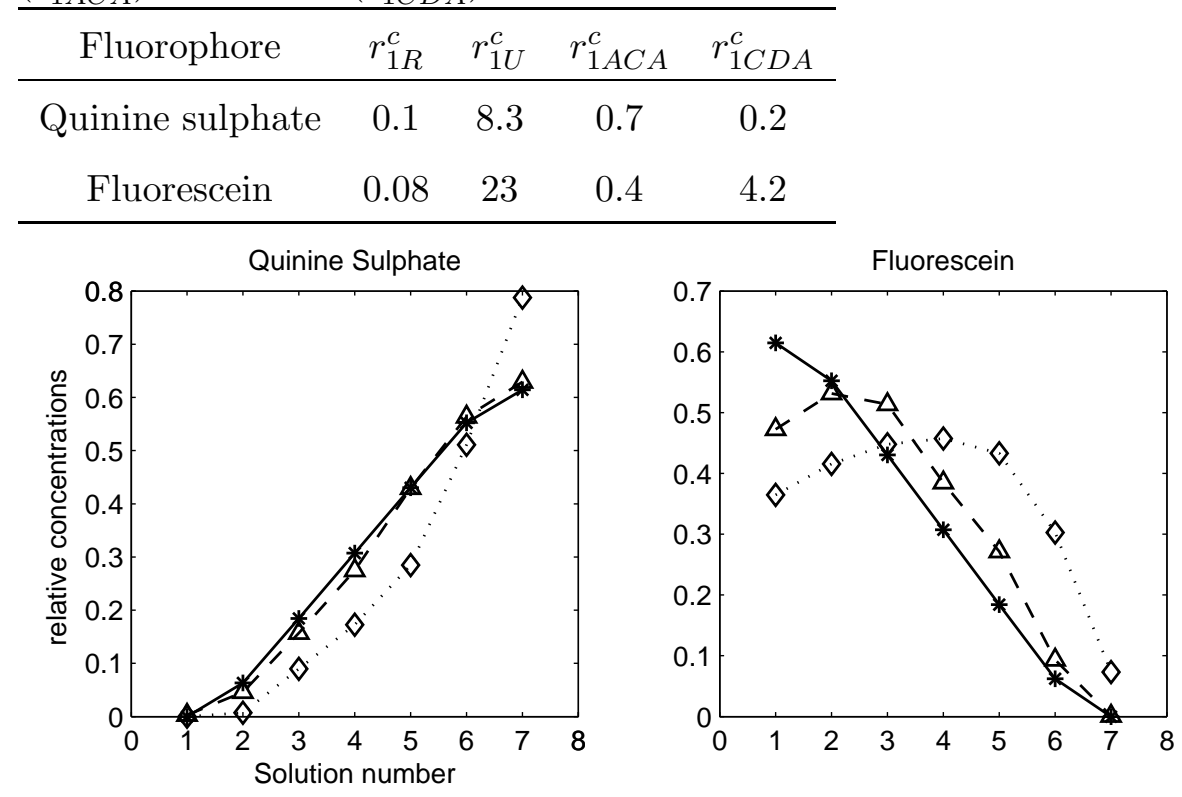

ACA-PARAFAC and CDA-PARAFAC are mainly due to inner filter effects and not to the PARAFAC decomposition.

Fig. 7. Data set 1, PARAFAC loadings of the concentration mode: Real spectra (solid $*$ line), U-PARAFAC loadings (dot $\diamond$ line) and CDA-PARAFAC loadings $($ dash $\triangle$ line $)$

Results for the concentration mode are presented in table 4 and figure 7 . The shape of the concentration profile of the quinine sulphate is slightly affected by filter effects $\left(r_{1 U}^{c}=8.3 \%\right)$. However CDA-PARAFAC gives much more accurate results for each solution $\left(r_{1 C D A}^{c}=0.2 \%\right)$. ACA-PARAFAC is also satisfying but it is not as efficient as CDA. The concentration profile of the fluorescein is more distorted $\left(r_{1 U}^{c}=23 \%\right)$. CDA-PARAFAC performs well $\left(r_{1 C D A}^{c}=4.2 \%\right)$ but the relative error is still high for the first solution. It should be noted that ACA-PARAFAC $\left(r_{1 A C A}^{c}=0.4 \%\right)$ do better than CDAPARAFAC. This last result is surprising because it is in contradiction with the five other loadings.

Results for the excitation mode are presented in table 5 and figure 8 . The excitation mode is the most affected by inner filter effects, with $r_{1 U}^{e x}$ values of $12.9 \%$ and $95.15 \%$ for quinine sulphate and fluorescein respectively. Considering figure 8, quinine sulphate excitation spectrum is widened and flattened by inner filter effects. These distortions are completely eliminated by CDA- 
Table 5

Data set 1, excitation mode results. Comparison with the real spectra among three criteria: relative error on the maximum value $(\%)$, shift $(\mathrm{nm})$ and relative squared error $(\%)$

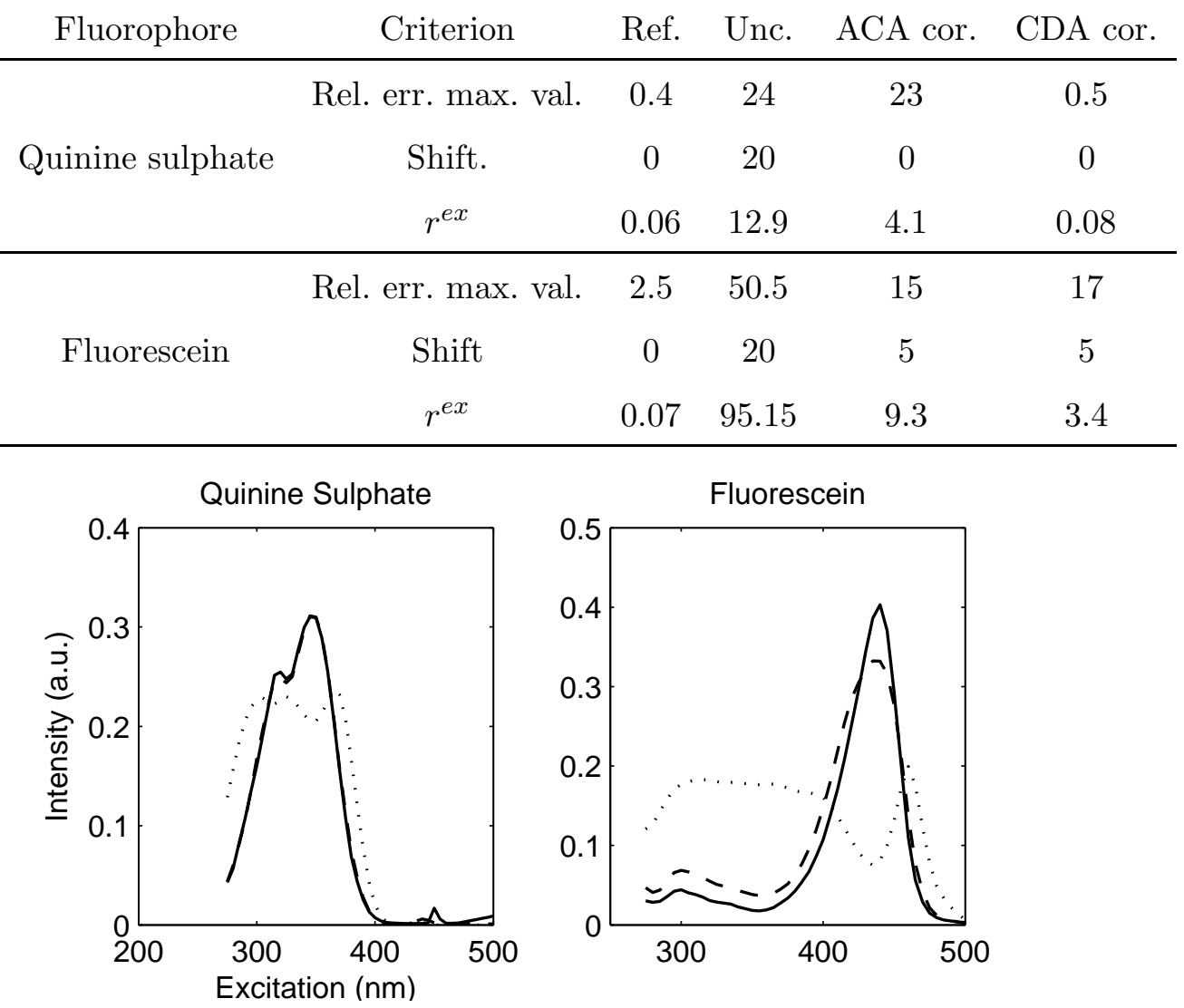

Fig. 8. Data set 1, PARAFAC loadings of the excitation mode: Real spectra (solid line), U-PARAFAC loadings (dot line) and CDA-PARAFAC loadings (dash line)

PARAFAC. Indeed, the CDA-PARAFAC estimated spectrum is really close to the real spectrum $\left(r_{1 C D A}^{e x}=0.08 \%\right)$. ACA-PARAFAC $\left(r_{1 A C A}^{e x}=4.1 \%\right)$ globally improves U-PARAFAC result $\left(r_{1 U}^{e x}=12.9 \%\right)$. The relative error on the maximum value is negligible with CDA-PARAFAC (0.5\%) while it as important with ACA-PARAFAC (23\%) as without correction (24\%). The $20 \mathrm{~nm}$ shift on the position of the maximum is perfectly corrected by both CDAPARAFAC and ACA-PARAFAC. These observations hold true for the fluorescein spectrum but the gaps between the different estimations are wider. The spectrum estimated by U-PARAFAC is totally distorted in respect to the real spectrum, $r_{1 U}^{e x}=95.15 \%$. In spite of some residual distortions (flattening and widening), the spectrum shape is almost fully recovered with CDA correction $\left(r_{1 C D A}^{e x}=3.4 \%\right)$. ACA-PARAFAC global estimation is not as good $\left(r_{1 A C A}^{e x}=9.3 \%\right)$. In absence of correction, the relative error on the maximum value is very high (50.5\%). ACA-PARAFAC and CDA-PARAFAC relative error are equivalent with $15 \%$ and $17 \%$ respectively. In the same way the 


\section{Table 6}

Data set 1, emission mode results. Comparison with the real spectra among three criteria: relative error on the maximum value $(\%)$, shift $(\mathrm{nm})$ and relative squared error $(\%)$

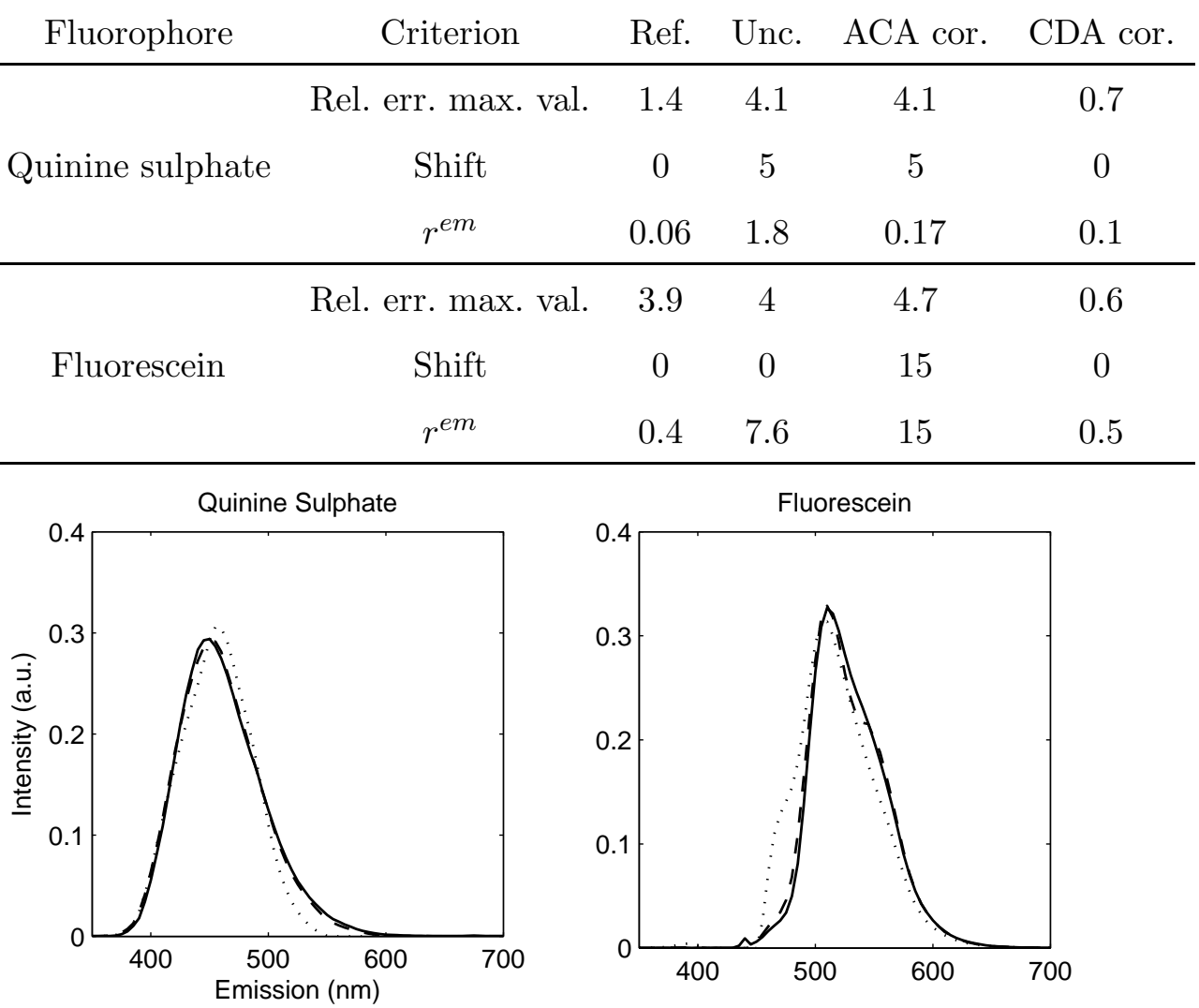

maximum shift is limited by ACA-PARAFAC and CDA-PARAFAC to the fluorometer excitation step (5 nm) against $20 \mathrm{~nm}$ with U-PARAFAC.

Fig. 9. Data set 1, PARAFAC loadings of the emission mode: Real spectra (solid line), U-PARAFAC loadings (dot line) and CDA-PARAFAC loadings (dash line) 
real limitation on this loading as the estimated spectrum is worse than the one estimated by U-PARAFAC regarding the three criteria (table 6). The relative squared error is twice higher (15\% against $7.6 \%)$ with ACA-PARAFAC than with U-PARAFAC while it is negligible with CDA-PARAFAC $(0.5 \%)$. The relative error on the maximum value is quite small with U-PARAFAC (4\%) and ACA-PARAFAC $(4.7 \%)$ but it is totally corrected by CDA-PARAFAC (0.7\%). Eventually, ACA-PARAFAC introduces a $15 \mathrm{~nm}$ shift on the position of the maximum which do not exist with U-PARAFAC and CDA-PARAFAC. In conclusion to this test, as expected, U-PARAFAC provides the worst results. These are very bad, specially for the fluorescein excitation spectrum and quinine sulphate emission spectrum whose the wavelength domains strongly overlap. ACA-PARAFAC improves these results. Regarding ACA results, loadings estimation is better than expected. However, it is outperformed by CDAPARAFAC at the exception of the fluorescein concentration profile. CDAPARAFAC results are indeed closer to those obtained with the reference FEEM although a larger error is observed on fluorescein excitation (table 5) and concentration loadings (table 6). This must be seen as the PARAFAC manifestation of the small distortion observed on the CDA correction of solution 1 (figure 6) and more generally this is an indication of the CDA limitations. Actually, CDA is limited to a certain domain of validation because equation 11 is obtained after several approximations. This holds true for any correction method relied on equation 11. Regarding the results of table 3 and figure 7 , this limitation has probably been reached with solution 1 . In these cases of very high absorbance (equal or above 2), more sophisticated models should be used. Nevertheless, we have demonstrated here that the validation domain of CDA is much larger than the linear one. Then the FEEM provided by CDA are close enough to the ideal linear FEEM to allow advanced spectral data analysis such as the PARAFAC decomposition while this is not the case with uncorrected FEEM or with ACA to a lesser extent. This example also show that CDA-PARAFAC improves both kinds of PARAFAC results: On the one hand it allows to recover the overall profile of strongly distorted loadings, on the other hand it provides some very accurate estimations of less affected loadings.

\subsection{Data set 2, application to field}

In this section, performances of CDA and CDA-PARAFAC are shown for the correction and the decomposition of mixtures of model molecules. The first stage of the test is a comparison between the reference FEEM $\left(I_{2 R}\right)$, the uncorrected FEEM $\left(I_{2 U}\right)$ and the CDA corrected FEEM $\left(I_{2 C D A}\right)$ of data set 2. Four representative examples of these different FEEM are presented on figure 10. Regarding these examples a main distortion appears on the fluorescence pattern if no correction is applied. It turns out that filter effects increase the 
peak around $(480 \mathrm{~nm}, 520 \mathrm{~nm})$ and decrease the intensity of the fluorescence signal located under $400 \mathrm{~nm}$ in excitation. This area is composed by several peaks which are partially recovered by CDA. The PARAFAC decompositions should show whether this finer aspect of the correction is satisfying or not. The relative squared residual error terms $\left(r_{2 U}\right.$ and $\left.r_{2 C D A}\right)$ of the whole data set are given in table 7. FEEM obtained with CDA are not as closed to the reference FEEM as for data set 1 . Actually, $r_{2 C D A}$ average value is about $4 \%$ while it is about $55 \%$ for $r_{2 U}$. Hence, it proves that CDA correction is still very beneficial relatively to the uncorrected FEEM.

Table 7

Comparison of the relative squared residual error terms (in \%) for the eleven solution of data set 2

\begin{tabular}{cccccccccccc} 
Sample & 1 & 2 & 3 & 4 & 5 & 6 & 7 & 8 & 9 & 10 & 11 \\
\hline$r_{2 U}$ & 51 & 66 & 35 & 59 & 35 & 44 & 83 & 76 & 83 & 10 & 60 \\
$r_{2 C D A}$ & 3 & 3 & 5 & 1.4 & 5 & 3 & 4 & 5 & 6 & 5 & 2 \\
\hline
\end{tabular}

The PARAFAC-ALS algorithm was applied to $I_{2 R}, I_{2 U}$ and $I_{2 C D A}$. In opposition to set 1 , the real number of fluorophore is unknown. This is actually the main problem with PARAFAC analysis of unknown FEEM. We compared the results provided by three classical tests: residual variance analysis, split half analysis and CORCONDIA. Finally, three components were used for the decompositions. These will be labelled fluorophore 1, 2 and 3 in the following. The real fluorophores are also unknown, consequently, R-PARAFAC loadings are taken as references for the evaluation of U-PARAFAC and CDAPARAFAC results. This comparison is made on tables 8 to 10 and figures 11 to 13 .

Table 8

Data set 2, concentration mode results. Relative squared residual error in percent between the reference loadings and U-PARAFAC loadings $\left(r_{2 U}^{c}\right)$ or CDA-PARAFAC loadings $\left(r_{2 C D A}^{c}\right)$.

\begin{tabular}{ccc} 
Fluorophore & $r_{2 U}^{c}$ & $r_{2 C D A}^{c} \cdot$ \\
\hline 1 & 6.6 & 5.6 \\
2 & 26 & 4 \\
3 & 7.3 & 8.2 \\
\hline
\end{tabular}

Results for the concentration mode are presented in table 8 and figure 11. Inner filters and CDA correction have little effects on the profile of fluorophore $1\left(r_{2 U}^{c}=6.6 \%\right.$ and $\left.r_{2 C D A}^{c}=5.6 \%\right)$ and $3\left(r_{2 U}^{c}=7.3 \%\right.$ and $\left.r_{2 C D A}^{c}=8.2 \%\right)$. On the opposite, fluorophore 2 is far more affected. The concentration profile estimated by U-PARAFAC is clearly unsatisfying $\left(r_{2 U}^{c}=26 \%\right)$. CDA-PARAFAC provides an acceptable estimation as the error term decreases to $4 \%$. Finally 

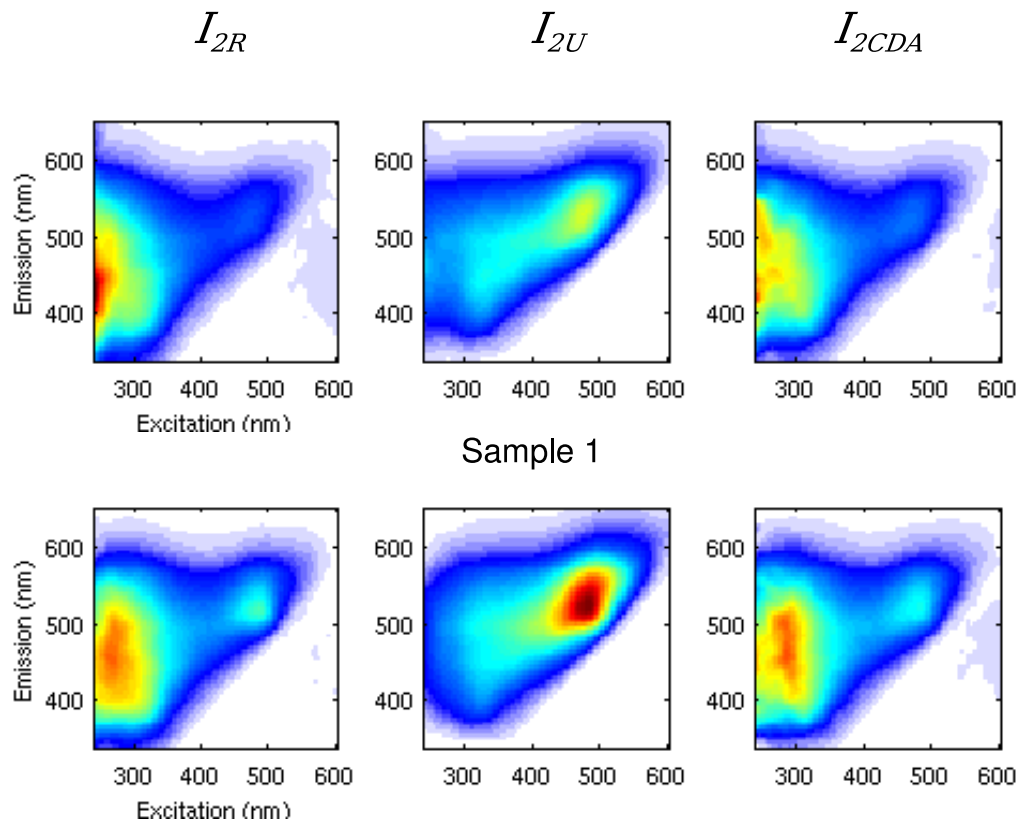

Sample 4
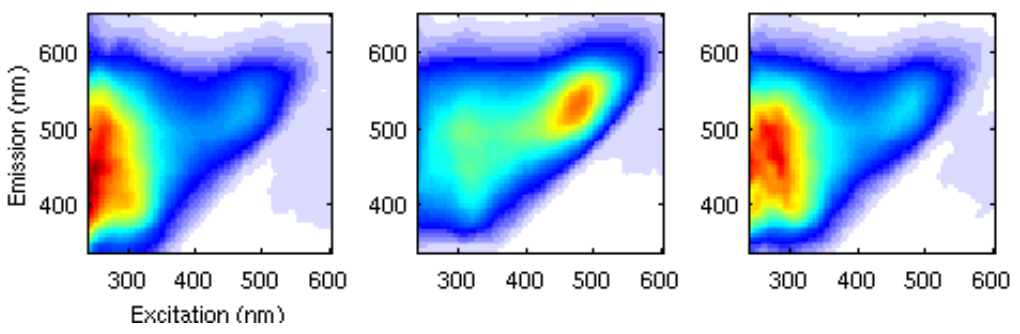

Sample 6
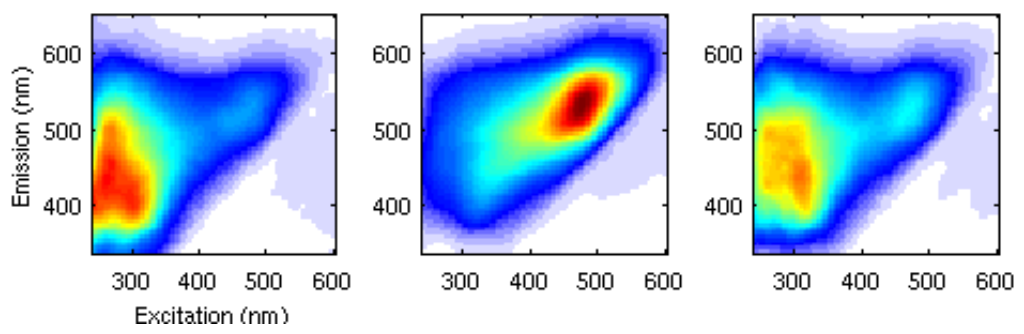

Sample 9

Fig. 10. Illustrations of $I_{2 R}, I_{2 U}$ and $I_{2 C D A}$ for samples 1, 4, 6 and 9 of data set 2 .

the three estimated profiles by CDA-PARAFAC are satisfying at the exception of sample 8 and 9. At the opposite of $r_{2 U}^{c}, r_{2 C D A}^{c}$ is greater for fluorophores 1 and 3. This is mainly due to the larger estimation errors on samples 8 and 9.

Results for excitation mode are presented in table 9 and figure 12. Excitation mode is the most affected mode of the decomposition as for data set 1 . Estimation of fluorophore 1 excitation spectrum takes a clear advantage of 

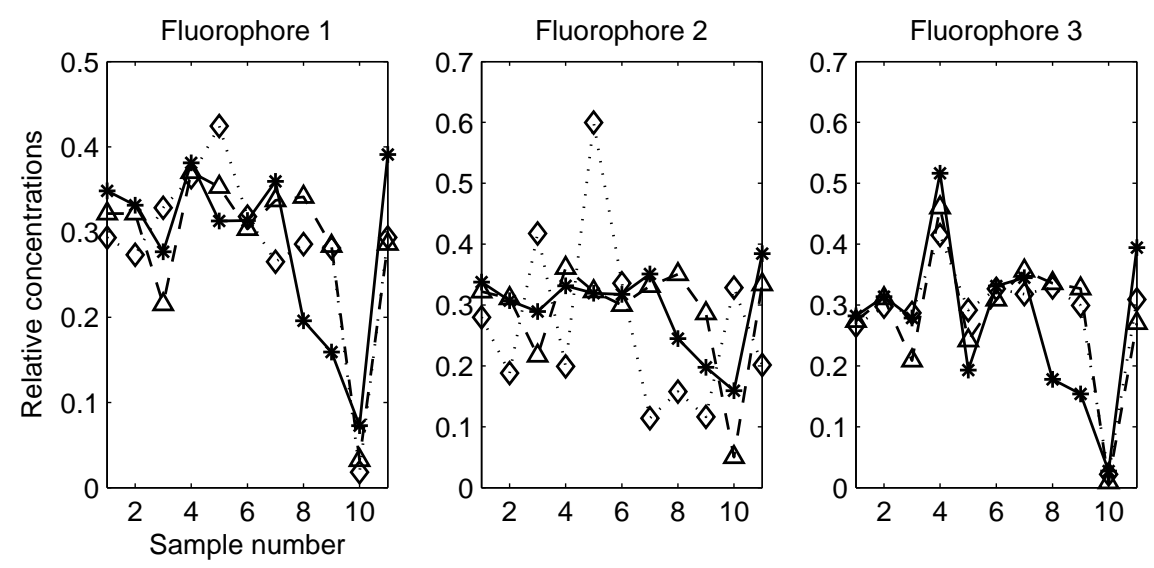

Fig. 11. Data set 2, PARAFAC loadings of the concentration mode: R-PARAFAC loadings (solid $*$ line), U-PARAFAC loadings (dot $\diamond$ line) and CDA-PARAFAC loadings (dash $\triangle$ line)

Table 9

Data set 2, excitation mode results. Comparison with the reference loadings among three criteria: relative error on the maximum value (\%), shift (nm) and relative squared error (\%)

Fluorophore Test Unc. CDA cor.

\begin{tabular}{cccc}
\hline & Rel. err. max. val. & 18 & 11 \\
1 & Shift & 185 & 15 \\
& $r^{\text {ex }}$ & 110 & 1.5 \\
\hline \multirow{2}{*}{2} & Rel. err. max. val. & 30 & 20 \\
& Shift & 20 & 0 \\
& $r^{e x}$ & 9.6 & 2.1 \\
\hline \multirow{2}{*}{3} & Rel. err. max. val. & 27.5 & 0.2 \\
& Shift & 10 & 5 \\
& $r^{\text {ex }}$ & 35 & 0.6 \\
\hline
\end{tabular}

CDA-PARAFAC. Regarding shape and position, the spectrum estimated by U-PARAFAC is a far cry from the reference spectrum $\left(r_{2 U}^{e x}=110 \%\right)$. On the opposite, CDA-PARAFAC provides a very good estimation $\left(r_{2 C D A}^{e x}=1.5 \%\right)$. The relative error on the maximum value is smaller (11\% against $18 \%$ ) but above all, the large shift $(185 \mathrm{~nm})$ is brought back to $15 \mathrm{~nm}$. Despite UPARAFAC estimation of fluorophore 2 spectrum is acceptable $\left(r_{2 U}^{e x}=9.6 \%\right)$, CDA-PARAFAC improves this result $\left(r_{2 C D A}^{e x}=2.1 \%\right)$. In absence of correction, the double peak disappears. CDA-PARAFAC correctly restores this feature but the relative error on the maximum value remains high (20\%). On the other hand, the $20 \mathrm{~nm}$ shift is completely corrected. U-PARAFAC estima- 

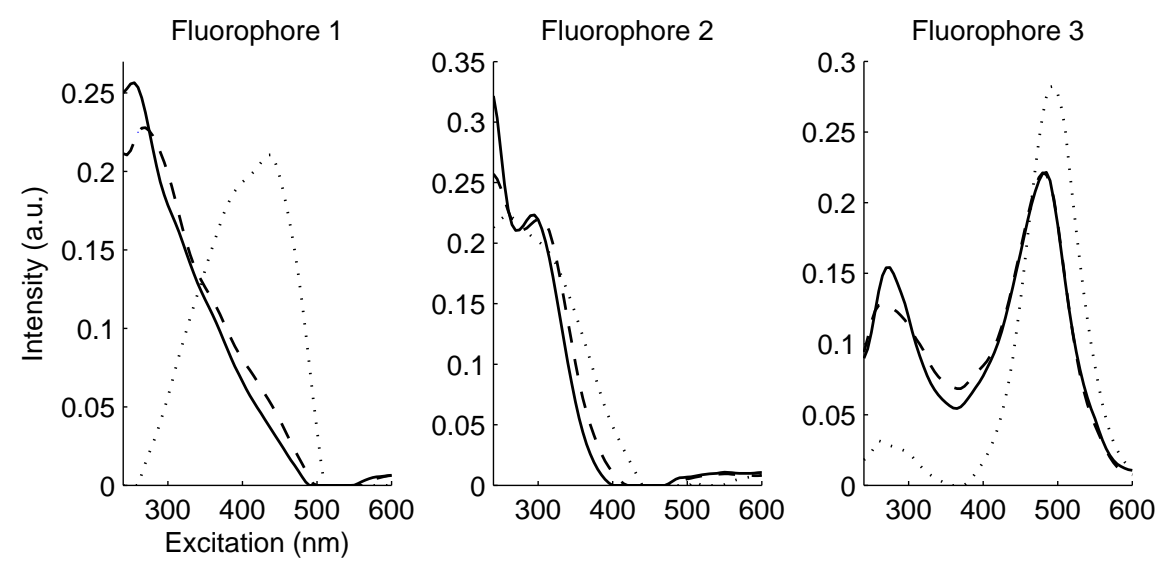

Fig. 12. Data set 2, PARAFAC loadings of the excitation mode: R-PARAFAC loadings (solid line), U-PARAFAC loadings (dot line) and CDA-PARAFAC loadings (dash line)

Table 10

Data set 2, emission mode results. Comparison with the real spectra among Comparison with the reference loadings among three criteria: relative error on the maximum value (\%), shift (nm) and relative squared error (\%)

\begin{tabular}{cccc} 
Fluorophore & Test & Orig. & Corr.Dil. \\
\hline \multirow{3}{*}{1} & Rel. err. max. val. & 13 & 12 \\
& Shift & 40 & 25 \\
$r^{e m}$ & 21 & 4 \\
\hline \multirow{2}{*}{2} & Rel. err. max. val. & 21 & 1.8 \\
& Shift & 60 & 10 \\
& $r^{e m}$ & 63 & 6 \\
\hline \multirow{3}{*}{3} & Rel. err. max. val. & 5.2 & 1.4 \\
& Shift & 20 & 10 \\
& $r^{e m}$ & 8.8 & 0.9 \\
\hline
\end{tabular}

tion of fluorophore 3 spectrum is not satisfying $\left(r_{2 U}^{e x}=35 \%\right)$. One of the two peaks almost disappears while the second one is overestimated. Nevertheless, its estimation by CDA-PARAFAC is almost identical to the reference spectrum $\left(r_{2 C D A}^{e x}=0.6 \%\right)$. The relative error on the maximum value is significant with U-PARAFAC $(27.5 \%)$ but it becomes negligible with CDA-PARAFAC $(0.2 \%)$. The shift is also reduced from $10 \mathrm{~nm}$ to $5 \mathrm{~nm}$. 

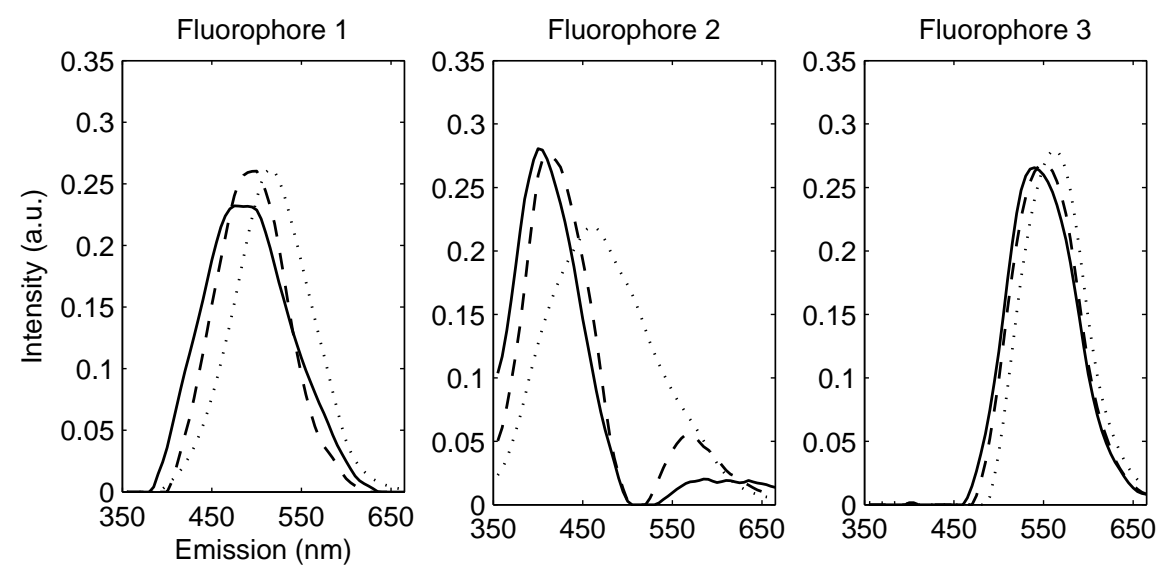

Fig. 13. Data set 2, PARAFAC loadings of the emission mode: R-PARAFAC loadings (solid line), U-PARAFAC loadings (dot line) and CDA-PARAFAC loadings (dash line)

value of $13 \%$ ) and CDA-PARAFAC (12\%). On the opposite, CDA-PARAFAC limits to $25 \mathrm{~nm}$ the large shift $(40 \mathrm{~nm})$ observed when no correction is applied. Fluorophore 2 spectrum is more severely affected by inner filter effects. CDA-PARAFAC provides a very satisfying estimation of this spectrum $\left(r_{2 C D A}^{e m}=6 \%\right)$ in respect to U-PARAFAC result $\left(r_{2 U}^{e m}=63 \%\right)$. The relative error to the maximum value observed with U-PARAFAC is important (21\%) but it is well corrected by CDA-PARAFAC (1.8\%). In the same way, the 60 $\mathrm{nm}$ shift is limited to $10 \mathrm{~nm}$. Fluorophore 3 spectrum is correctly estimated by U-PARAFAC $\left(r_{2 U}^{e m}=8.8 \%\right)$. CDA-PARAFAC still improves the estimation $\left(r_{2 C D A}^{e m}=0.9 \%\right)$. The relative error to the maximum value is lower $(1.4 \%$ against $5.2 \%$ ) and the shift is reduced from $20 \mathrm{~nm}$ to $10 \mathrm{~nm}$.

CDA-PARAFAC results on data set 2 are also conclusive. All the loading are indeed correctly estimated. Relative concentrations of only one fluorophore in only two samples out of eleven are poorly estimated and all the estimated spectra are close enough to the corresponding reference spectra. The crucial point on this second example is that in a real case situation of DOM tracing, CDAPARAFAC would probably give interpretative results while the uncorrected FEEM would provide misleading estimations. Moreover, excitation spectra of fluorophores 1 and 3 illustrate the two main kinds of spectral deviations due to inner filters. Fluorophore 1 shape is distorted and its position is shifted from $275 \mathrm{~nm}$ to $480 \mathrm{~nm}$. On the opposite, the position of fluorophore 3 is unchanged, its global shape is almost correct but the respective magnitude of its two peaks is largely modified. CDA-PARAFAC provides an impressive correction of both deviations.

Actually, CDA-PARAFAC appears to be a critical improvement of U-PARAFAC or even ACA-PARAFAC in the case of strong inner filter effects. 


\section{Conclusion}

It is possible to correct inner filter effects by using simply a controlled dilution approach (CDA). This analytical solution is better than usual absorbance correction and quicker and safer than strong dilution, under absorbance of 0.3. It has been demonstrated in this work the good ability of CDA and CDAPARAFAC in the case of standard mixtures of two fluorophores and in the case of real DOM samples. In this study, CDA performed very well for solution absorbances up to 1.83. Further investigation should be made outside this range. We conjecture that an other theoretical model of fluorescence measurement should be used for absorbance higher than 2 .

In respect to the PARAFAC decomposition, better results were obtained on the spectral loadings. We have also highlighted the limit of ACA for the correction of strong filter effects. Consequently, we recommend the use of CDA for FEEM experiment and PARAFAC pretreatment to avoid error and misinterpretation.

\section{References}

[1] B. Valeur, Molecular Fluorescence. Principles and Applications, Wiley-VCH, Weinheim, 2002.

[2] A. Hansch, D. Sauner, I. Hilger, J. Böttcher, A. Malich, O. Frey, R. Bräuer and W.A. Kaiser, Academic Radiology, 11 (11) (2004) 1229-1236.

[3] E. Sikorska, T. Gorecki, I.V. Khmelinskii, Sikorski and D. de Keukeleire, Food chemistry, 96 (4) (2006) 632-639.

[4] G.P., Coble, Marine Chemistry, 51 (1996), 325-346.

[5] C.A. Stedmon, S. Markager, Limnology and Oceanography, 50 (2) (2005).

[6] J.R., Lakowicz, Principles of Fluorescence Spectroscopy, Plenum Press, NY, 1983.

[7] C.A. Parker, W.J. Barnes, The analyst, 82 (1957) 606-618.

[8] J.J. Mobed, S.L. Hemmingsen, J.L. Autry, and L.B. McGown, Environmental Science and Technology, 30 (10) (1996) 3061-3065.

[9] J.F. Holland, R.E. Teets, P.M. Kelly and A. Timnick, Analytical Chemistry, 49 (6) (1977) 706-710.

[10] C. M. Yappert and J.D. Ingle, Applied Spectroscopy, 43 (5) (1989) 759-767.

[11] S.A. Tucker, V.L.Amszi, W.E. Acree, Journal of Chemical Education, 69 (1992). 
[12] B.C. MacDonald, S.J.Lvin and H. Patterson, Analytica Chimica Acta, 338 (1997) 155-162.

[13] A. Credi, and L. Prodi, Spectrochimica Acta Part A, 54 (1998) 159-170.

[14] J. Riesz, J. Gilmore, P. Meredith, Spectrochimica Acta part A (2004).

[15] T. Ohno, Environmental Science and Technology, 36 (4) (2002) 742-746.

[16] D.M. MacKnight, E.W Boyer, P.K. Westerhoff, P.T. Doran, T. Kulbe and D.T. Andersen, Limnology and Oceanography, 46 (2001) 38-48.

[17] F. Cuesta Sanchez, S. Rutan, M. Gil Garcia and D. Massart, Chemometrices and Intelligent Laboratory Systems, 36 (1997) 153-164.

[18] A. Garrido Frenich, D. Picon Zamora, J. Martinez Vidal and M. Martinez Galera, Analytica Chimica Acta, 449 (2001) 143-155.

[19] J. Boehme, P. Coble, R. Conmy and A. Stovall-Leonard, Marine Chemistry, 89 (2004) 3-14.

[20] J.M. Andrade, M.P. Gomez-Carracedo, W. Krzanowsky and M. Kubista, Chemometrices and Intelligent Laboratory Systems, 72 (2004) 123-132.

[21] R.A. Harshman, UCLA Working Papers in Phonetics, 16 (1970) 1-84 (UMI Serials in Microform, No. 10,085).

[22] R. Bro, Chemometrics and Intelligent Laboratory Systems, 38 (1997) 149-171.

[23] C.A. Stedmon, S. Markager and R. Bro, Marine Chemistry, 82 (3-4) (2003) 239-254.

[24] R.D. Holbrook, J.H. Yen and T.J. Grizzard, Science of The Total Environment, 361 (1-3) (2006) 249-266.

[25] X. Luciani, S. Mounier, H.H.M. Paraquetti, R. Redon, Y. Lucas, A. Bois, L.D. Lacerda, M. Raynaud and M. Ripert, Marine Environmental Research, 65 (2) (2008) 148-157.

[26] D. Baunsgaard, Department of Dairy and Food Science, The Royal Veterinary and Agricultural University, (1999).

[27] G.G. Anderson, K.D. Brian and K.S. Booksh, Chemometrics and Intelligent Laboratory Systems, 49 (1999) 195-213.

[28] O. Divya and A.K. Mishra, Analytica Chimica Acta, 630 (2008) 47-56.

[29] P. John, I. Soutar, Analytical Chemistry, 48 (1976) 520-524.

[30] D. Patra and A.K. Mishra, Analyst, 125 (2000) 1383-1386

[31] R.A. Harshman, UCLA Working Papers in Phonetics, 22 (1972) 111-117 (UMI Serials in Microform, No. 10,085).

[32] N.D. Sidiropoulos and R. Bro, Journal of Chemometrics, 14 (3) (2000) 229-239. 
[33] N.M. Faber, R. Bro and P.K. Hopke, Chemometrics and Intelligent Laboratory Systems, 65 (2003) 119-137.

[34] G. Tomasi, R. Bro, Computational Statistics \& Data Analysis, 50 (7) (2006) 1700-1734.

[35] P. Paatero, Chemometrices and Intelligent Laboratory Systems, 38 (2) (1997) 223-242.

[36] R.A. Harshman and M.E. Lundy, Computational Statistics \& Data Analysis, 18 (1994) 39-72.

[37] C. Andersen, R. Bro, Journal of Chemometrics, 17 (2003) 200-215.

[38] R.G. Zepp, W.M. Sheldon and M.A. Moran, Marine Chemistry, 89 (2004) 15-36.

[39] C.A. Andersson and R. Bro, Chemometrics and Intelligent Laboratory Systems, 52 (2002) 1-4.

[40] R. Bro and H.A.L. Kiers, Journal of Chemometrics, 17 (2003) 274-286. 